\title{
Towards A New Opportunistic IoT Network Architecture for Wildlife Monitoring System
}

\author{
Eyuel D. Ayele, Nirvana Meratnia, Paul J.M. Havinga \\ Pervasive Systems Research Group \\ University of Twente, Enschede, the Netherlands \\ Email: \{e.d.ayele, n.meratnia, p.j.m.havinga\}@utwente.nl
}

\begin{abstract}
In this paper we introduce an opportunistic dual radio IoT network architecture for wildlife monitoring systems (WMS). Since data processing consumes less energy than transmitting the raw data, the proposed architecture leverages opportunistic mobile networks in a fixed LPWAN IoT network infrastructure. This solution will facilitate an IoT devices to be deployed for ultra-low power and sustainable wildlife monitoring applications. As part of the IoT infrastructure, a LoRa based network is presented with coverage characterization and preliminary test bed deployment for wildlife tracking purpose. In addition, through simulation, the utilization of existing BLE based opportunistic data collection protocols for the proposed architecture is investigated.
\end{abstract}

Keywords-LPWAN, LoRa, Internet of Things, IoT, BLE, Network Architecture, Opportunistic networks

\section{INTRODUCTION}

For the past few years, utilization of Internet of Things (IoT) have grown steadily. Wildlife monitoring is one of the trending applications of IoT technologies, where a number of heterogeneous sensors are deployed to monitor the activities of wild animals dwelling in a remote and geographically large habitat. It is known that wild animals depict similar physiological activities as a herd [1]. Thus, a number of heterogeneous sensors (e.g. accelerometer, gyroscope, etc) could be deployed either as collars or buried in the ground, to monitor the physiological activities. The sensors will be operating in a remote area with a limited source of energy. To these ends, it is important to achieve high energy efficiency, good reliability and low latency for a responsive wildlife monitoring system (WMS) design. WMS deployment facilitates the local data pre-processing and sharing among end-nodes for collaborative decision making. Data processing consumes less energy than transmitting it. Thus, instead of reporting all the raw data generated from the end-devices, WMS performs local preprocessing and relay the aggregated data at a reduced energy consumption to the central server [2].

In the past, wireless sensor networks with conventional routing algorithms are used, (e.g DSR or AODV [3]), but they often perform poorly in scenarios where the communication path is intermittent due to animal mobility as in WMS. Opportunistic mobile networks (OMNs) are the recent evolution of the traditional mobile wireless sensor networks (MWSN), which are used to provide communication facilities among devices in sparse and mobile network scenarios, similar to wildlife monitoring applications. Several opportunistic data dissemination schemes, on sensor data gathering, have been proposed in a number of recent studies, e.g. Epidemic, Spray and Wait (SnW), Direct Contact (DC), etc. [4]. OMNs have been implemented successfully in various animal monitoring projects e.g. ZebraNet, Rat Watch [5]. These networks are developed on top of IEEE802.15.4 standard, which often suffers from huge overheads due to the complex implementation [7]. More recently, the energy efficient version of Bluetooth known as $B L E$ - Mesh, has better energy efficiency and wider coverage over IEEE802.15.4 based solutions [8]. While making some progress in energy efficiency aspect, these solutions still suffer from high latency mainly due to node mobility.

Although this issue can be addressed by using complementary long range wireless technologies (e.g., cellular) to facilitate connectivity, they often consume higher power. In addition, remote areas where wild animals dwell are often out of cellular coverage. Fortunately, emerging low power wide area network (LPWAN) technologies such as LoRa, Sigfox, etc. promise to provide better coverage with a low energy consumption that seem to support many requirements of remote wildlife monitoring application [11]. LPWANs in general are fundamentally designed to ensure very long battery lifetime and provide seamless interoperability among enddevices without the need for complex local installations. Among LPWAN technologies, LoRa is believed to have high potential for realization of LPWAN IoT goals. However, IoT applications requiring high data rate and low latency are not particularly the strength of LPWANs mainly due to the generic low bit-rate, stricter duty-cycle restrictions and the larger packet header associated with it. Thus to realize the WMS design requirements, a mechanism to control the tradeoff between energy versus latency is necessary, which is not practically achievable by using only LPWAN technology [11]. Although few works have been conducted to address this aspect by proposing an architecture for WMS, to the best of authors' knowledge, none of these works integrate OMN and LPWAN infrastructure in their approach.

Therefore, in this paper we propose a WMS network architecture by utilizing LPWAN and opportunistic mobile networks $(\mathrm{OMN})$. The results and analysis presented in this paper are set-up by considering LoRa as an LPWAN technology and BLE as an enabler for opportunistic network. This type of architecture can find use in wildlife scenarios where LoRa can act as collection stations, and collared BLE sensor nodes 
as regular sources that generate and concatenate data in the network. To this end, the contributions of this paper are:

- Propose a new IoT network architecture for wildlife monitoring system along with preliminary prototype deployment of animal tracker.

- Investigate the performance of opportunistic BLE network with existing data dissemination protocols in terms of delivery ratio and average latency.

The rest of the paper is organized as follows: Section II presents the proposed network architecture. Section III discusses the opportunistic BLE networking and evaluation results. Section IV presents the LoRa IoT infrastructure network. Finally, Section $\mathrm{V}$ outlines the concluding remarks and future research challenges.

\section{WildLIFE MONITORING SYSTEM (WMS)}

\section{A. Overview of LoRa and BLE}

LoRa physical layer, developed by Semtech [14], allows for long-range, low-power and low bit rate communications. The operating frequency of LoRa are $433 / 868 / 915 \mathrm{MHz}$ depending on the country it is deployed. LoRa radio link is based on a proprietary chirp spread spectrum modulation scheme. Depending on the LoRa radio configuration the data payload size can reach from 2 to 255 bytes, and the bit-rate could reach $5.4 \mathrm{kbps}$ when configured at the highest bit-rate setting. The LoRaWAN version 1.0 specification, steered by LoRa Alliance, proposes open standard LoRaWAN as the MAC layer which targets to support mobile end-devices with uplink and downlink data communication. LoRa radio modulation itself can be used in different network topologies. However, the LoRaWAN architecture is configured as a star-of-star topology, it basically includes three components, i.e. end-devices, LoRa gateway, and a LoRa network server. LoRaWAN gateway are able to forward the control signals and froward data between a central network server and end-nodes. They are able to decode multiple signals at the same time.

Recently, Bluetooth Low Energy (BLE), also know as "Bluetooth Mesh", has surfaced as an appealing alternative, with many competing advantages over available low-power communication technologies in the IoT sphere such as IEEE 802.15.4. BLE is the light-weight and low power version of classical Bluetooth technology [8]. It operates in the $2.4 \mathrm{GHz}$ ISM band spectrum range utilizing a GFSK modulation. In theory BLE can reach up to $200 \mathrm{~m}$ in an open flat rural environment at maximum transmission power setting. Compared to IEEE 802.15.4 solutions, BLE offers a relatively higher data rate (upto $1 \mathrm{Mbps}$ ) and a low latency (typically $6 \mathrm{~ms}$ ) suitable for WMS requirements. The power efficiency of BLE makes it suitable for applications that run for longer periods on power sources, such as coin cell batteries or energyharvesting devices, as in wildlife monitoring scenarios. Neighbor discovery is one of the key mechanisms of PHY/MAC to efficiently administer self-organizable mobile networks. Unlike other short-range wireless technologies, e.g. WiFi direct, the recently released specification for BLE - Mesh v1.0 offers low-power and simple device discovery which easily enables opportunistic network deployment in WMS [9]. Despite these appealing features to WMS IoT application, BLE is a new standard thus it is still open to research and development for WMS implementation.

\section{B. Proposed Opportunistic IoT Network Architecture}

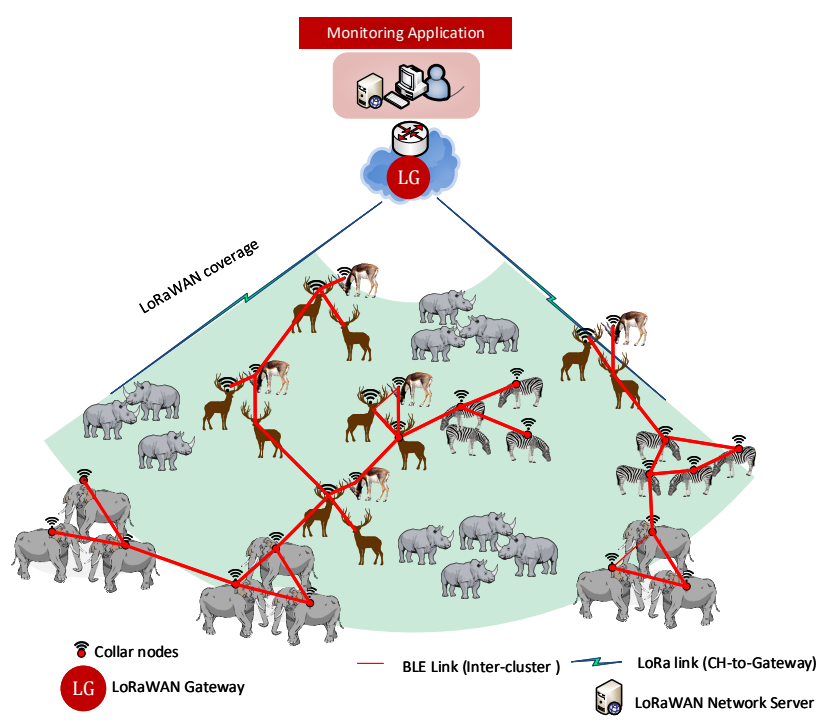

Fig. 1: Opportunistic IoT network architecture for wildlife monitoring system (WMS).

The proposed network architecture integrates LoRa IoT infrastructure and opportunistic mobile sensing as shown in Figure 1. In this paper, wild animals are assumed to be collared with inbuilt sensors and a dual radio platform (i.e. BLE and LoRa). The end-devices use short range BLE radio, for peerto-peer communication and long range LoRa radio to link to LoRa gateway. Thus, the proposed network have two main components: (i) BLE based opportunistic sensing and (ii) LoRa IoT infrastructure for facilitating connectivity. This type of mixed network architecture enables local pre-processing and data sharing among nodes for collaborative decision making. Moreover, instead of reporting all the raw data generated from the end-devices, WMS performs local pre-processing and relays the concatenated data at a reduced energy consumption to the central server [2]. LoRa network server runs the operation and management application, and run-time event monitoring, e.g. location tracking and mapping. There are two types of nodes in the BLE opportunistic network: (i) source node, which is responsible for generating data; (ii) sink node, which is able to translate and concatenate data between the opportunistic network and LoRa gateway (LG). This concatenation algorithm is out of scope for this paper, however, we plan to detail the procedure in future work. A source node sends data to sink node by utilizing an opportunistic - store-carry-forward (SCF) scheme. The nodes, except the LoRa gateway (LG), continuously forwards data when in communication range of other nodes. This opportunistic flooding approach to relay data 
best meets the requirements for enabling simple, reliable and scalable mesh networks in wild animal monitoring scenario.

\section{BLE BASED OpPORTUNISTIC SENSING}

This section investigates the proposed BLE based opportunistic network for wildlife monitoring applications.

\section{A. Simulation Set-up}

The evaluation set-up is similar to the one used in Ayele et al. [15] with additional parameter configurations. In addition we consider two mobility models. The first is random way point (RWP) movement in which a mobile node moves in random trajectory and at node speed range of $[10,30 \mathrm{~km} / \mathrm{h}]$ with $\max$-pause $=5 \mathrm{~s}$. Naturally, animals do not just wander around randomly, they prefer to go somewhere for a purpose, often using the fastest path possible [5]. These destinations are driven by diverse reasons, ranging from ordinary points of interest (e.g. water, grazing, etc.); to a more distressing activity (e.g. preying, running, etc.). Thus, we imported a second movement model from the ZebraNet GPS data to model the behavior of real animal mobility [6]. We evaluated the performance of the proposed opportunistic network in ONE simulator with the imported movement models [16].

The network is set-up as shown Figure 1. The MAC/PHY layers are abstracted to transmission range and data rate in the simulator. since our proposed architecture considers BLE interface for short-range communication; all mobile nodes have BLE radio at $250 \mathrm{kbps}$ data rate with $200 \mathrm{~m}$ radio range. Based on various animal species' empirically modeled data, it is known that the optimal average group or herd size is in the range of $n=[1,400]$. For instance, impala and zebra has a mean herd density of $\leq 70 / \mathrm{km}^{2}$ [17]. In both mobility models we simulate animals moving in a defined trajectory in a grid area of $10000 \mathrm{mx} 10000 \mathrm{~m}$. The network is set-up to run in a varying message generation rate $\left(T^{+}{ }_{m g}(\mathrm{sec}).\right)$. The BLE source nodes randomly generate data with 64 bytes payload at $\left(25 \leq T^{+}{ }_{m g} \leq 45\right)$. We remark that 64 bytes suffice for recording animal activity features (e.g. type and duration of activity), such as running, grazing, or walking. Table I summarizes the simulation parameters set.

TABLE I: Generic Input Simulation Parameters

\begin{tabular}{|ll|}
\hline Grid area & $10000 \times 10000 \mathrm{~m}^{2}$ \\
\hline Mobility model & RWP and ZebraNet \\
\hline Mobility speed & RWP $[10,30 \mathrm{Km} / \mathrm{h}]$ \\
\hline Simulation duration & $14 \mathrm{hr}$ \\
\hline RWP pause time & 5 Seconds \\
\hline Data rate (DR) & BLE $(250 \mathrm{kbps})$ \\
\hline Message TTL (min) & {$[0,30,60,120,300,480,700]$} \\
\hline$T^{+}{ }_{m g}($ sec. $)$ & $\left(25 \leq T_{m g} \leq 45\right)$ \\
\hline Tx range & BLE $(200 \mathrm{~m})$ \\
\hline Number of nodes & {$[1,400]$} \\
\hline Packet (PL) & 64 bytes \\
\hline Buffer size & $24 \mathrm{~kb}$ \\
\hline
\end{tabular}

In this work we only consider data to be generated from all source nodes towards one BLE sink node initially located at the center of grid area, however, through time all nodes move based on the mobility trajectory model supplied. We recorded the packet generation time as well as the time when they are received at the sink node. Sink node will concatenate data received and relay to the LoRa gateway. Furthermore, the BLE based opportunistic network is investigated under the benchmark opportunistic protocols, i.e. Direct Delivery, First Contact, Spray and Wait (SnW), Epidemic, and ProPHET. We evaluate the performance of the network against two metrics.

- Average latency: the difference between the time a data is sent at the source and time it is delivered at the destination (sink).

- Delivery ratio: the ratio of successfully delivered data to sink to total number of data transmitted by source nodes.

\section{B. Result Analysis}

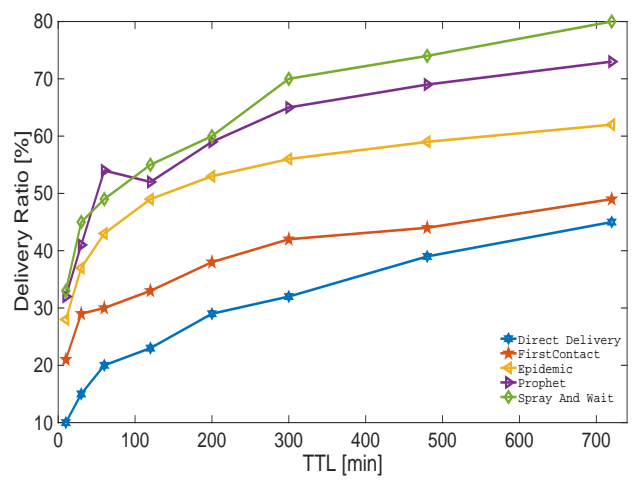

(a) RWP

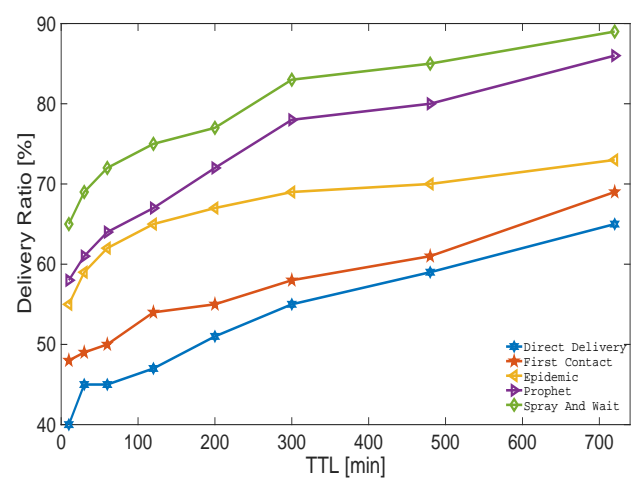

(b) ZebraNet

Fig. 2: Delivery Ratio for RWP and ZebraNet mobility models at $\left(25 \leq T^{+}{ }_{m g} \leq 45\right)$

In monitoring applications such as WMS, events are relayed with high priority. In this aspect, one of the influential network parameters is time-to-live (TTL) and it highly impacts network delivery ratio and latency. However, it is not throughly studied in previous works [4]. Figure 2 shows that, an increase in TTL of a data improved the trend of delivery ratio in both mobility cases. High TTL value is expected to increase the overall 


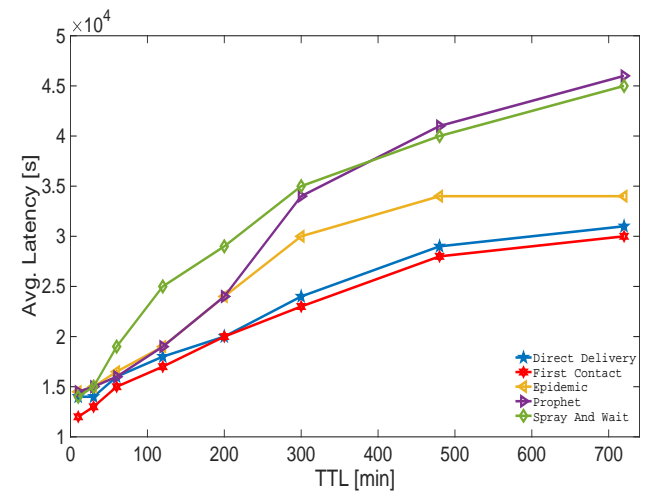

(a) $R W P$

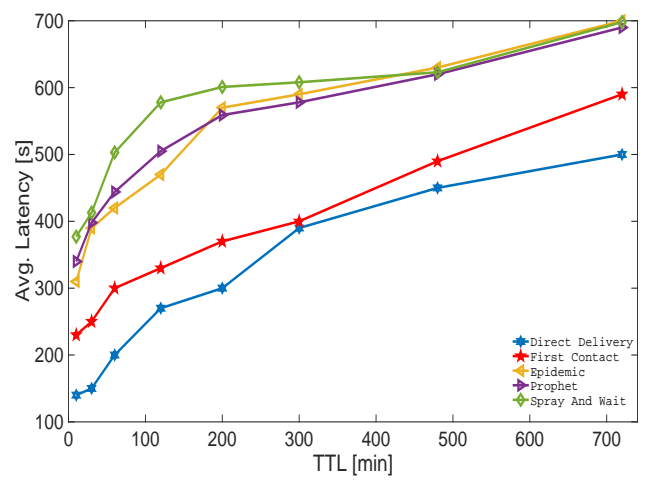

(b) ZebraNet

Fig. 3: Average Latency for RWP and ZebraNet mobility models at $\left(25 \leq T^{+}{ }_{m g} \leq 45\right)$

delivery ratio and vise versa [4]. The benchmark protocols in particular, shows a relatively high delivery ratio in case of ZebraNet for the specified range of $T^{+}{ }_{m g}$. DD results in a lower delivery ratio due to the allowed one copy of data to be made. Where the minimum observed delivery ratio using DD protocol is $10 \%$ and $40 \%$ respectively for RWP and ZebarNet mobility models. In general, FC, Epidemic, PRoPHET and SnW show a trend of increasing delivery ratio with TTL value. This is as expected, since for higher TTL the chance of data reaching the intended destination would be higher for highly mobile scenarios. This is mainly attributed to inherent data replication properties of routing protocols. In particular, SnW and PRoPHET are multi-copy, thus the number of duplicated data increases exponentially with higher TTL [4]. In general, since data is replicated to all nodes in a network, the overall storage requirements for probabilistic routing becomes high, reducing the routing performance. However, probabilistic routers e.g. SnW shows a maximum delivery ratio of approximately $80 \%$, which is higher than the DD or FC. Therefore, probabilistic routing is more suitable for wildlife opportunistic sensing as in WMS scenario.

Furthermore, Figure 3 shows the latency against TTL for both mobility models with $T^{+}{ }_{m g}$. Generally, the plot depicts that as TTL increases the overall data latency shows an increasing trend, this is in accordance with the previous works [4]. Although DD have the lowest delivery ratio at high TTL, DD shows shortest average delay, indicating an average latency of $\approx 130$ s and $\approx 1000$ s respectively for ZebarNet and RWP mobility models. This is mainly because DD delivers data directly to the sink when it is in range than relaying it through neighboring nodes. Generally, FC, Epidemic, SnW and ProPhet have similar trend of average latency as in shown in Fig. 3. This is due to the replication of data based on probability of contact, resulting in unnecessary flooding of data through out the entire network until it is received at sink node. Furthermore, the ZebraNet node movement shows shorter average latency than the RWP model, supporting the notion that opportunistic networks are applicable to wild life monitoring scenarios (Fig. 3).

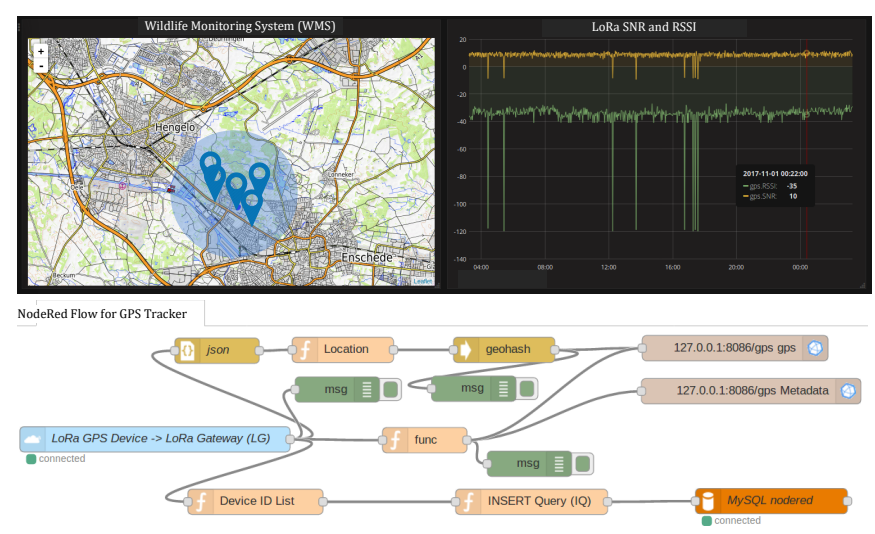

Fig. 4: WMS location tracking application on the LoRa server, a map showing near Univ. Twente Campus

\section{Preliminary LoRa IoT Testbed Deployment}

This section introduces an overview of LoRa radio characterization in a preliminary LoRa IoT test bed deployment. We built a LoRa radio with GPS capability using Adafruit Feather 32u4 RFM95 - 868MHz - module, equipped with an antenna. The LoRa gateway is deployed at one of the buildings of the University of Twente campus, where our test took place. We used LORANK as the receiving LoRa gateway. The gateway is programmed to listen for incoming packets on the $868 \mathrm{MHz}$ band. We set up a local light-weight runtime server built on Node.js, with NodeRed flow web application, as the server-side of LoRa network server (Fig. 4). LoRa end-devices send a periodically continues GPS location beacon to LoRa gateway (LG). We implemented Mysql and Influx databases to collect the transmitted packets from the LoRa device. While performing the test for several hours, we analyzed and visualized various real-time information about the received packets such as RSSI, SNR and Geohash location values to exactly locate and monitor the LoRa enabled trackers as shown in Figure 4. Thus, by utilizing LoRa enabled GPS sensors, 
the preliminary test showed that, we can provide a promising and cost effective solution for animal monitoring and tracking system. In the future, we plan to build a special collar prototype with an embedded BLE and LoRa-GPS trackers to deploy it in a real game parks.

\section{CONCLUSION AND WMS GUIDELINES}

In this paper, we presented a wildlife monitoring system (WMS), in the process we have isolated issues to consider while designing an apt WMS. A high delivery ratio, low latency and low energy are the main measures to provide real-time wildlife monitoring services. Moreover, providing large coverage area is an important aspect for a WMS. Data pre-processing locally consumes less energy, thus, instead of reporting all the raw data generated from the end-devices, WMS performs local processing and send the concatenated data at a lower energy consumption to LoRa server. In our proposed solution, a WMS is deployed with devices placed on animals or in the field, unattended, for months or years to come. Hence, each device has to have a low power functionality in addition to its local power supply in order to maximize the total system life-time in the long run. For applications requiring high network life-time at high packet traffic intensities, a combination of opportunistic BLE sensing and LoRa IoT infrastructure is more applicable. Table II summarizes the design guidelines for WMS network. Moreover, BLE easily integrates with existing LPWAN IoT technologies. LPWAN solutions (e.g. LoRa) are suitable for providing a larger coverage range (up-to $15 \mathrm{~km}$ ) at lower data rate, lower packet traffic intensities and low energy consumption which is ideal for relaying concatenated data from the sink node of BLE mesh network to LoRa server. Hence, in sparsely mobile sensor node distribution, as in a herd of con-specific animal population, leveraging BLE network with LoRa, could provide the required WMS deployment. Several well-known opportunistic protocols were tested with this architecture. It is observed that mobile nodes are best served by stochastic opportunistic schemes. Following this preliminary work, a testbed implementation is planned to validate the proposed complete heterogeneous network architecture including the BLE mesh protocol. We will present the complete network deployment in our future work.

TABLE II: Wildlife Monitoring System (WMS) guidelines

\begin{tabular}{|c|c|c|c|}
\hline & BLE & LoRa & $\begin{array}{l}\text { Proposed WMS } \\
\text { (LoRa + BLE Sensing) }\end{array}$ \\
\hline Topology & Mesh/star & $\begin{array}{l}\text { Star-of-star } \\
\text { (LP-WAN) }\end{array}$ & $\begin{array}{l}\text { Star-of-Mesh } \\
\text { (LP-WAN) }\end{array}$ \\
\hline Number of nodes & Unlimited & $100000 \mathrm{~s}$ & Unlimited \\
\hline (bytes) & 30 & 256 & 30 (mesh), 256 (LoRa) \\
\hline Throughput & High (250kbps) & Low (5.4kbps) & High \\
\hline Coverage-area & $\begin{array}{l}\text { Short }(\leq 200 \mathrm{~m}) \\
\text { (large with mesh) }\end{array}$ & Large $(\leq 15 \mathrm{~km})$ & Large \\
\hline Network lifetime & High & Low & High \\
\hline
\end{tabular}

\section{ACKNOWLEDGMENT}

This paper has been accepted for publication in the Workshop on Wireless Sensor Networks: Architectures, Deployments (WSN-ADT'2018). The research was supported by Smart Parks Project, funded by the Netherlands Organization for Scientific Research (NWO).

\section{REFERENCES}

[1] W. E. Cooper Jr. Escaping from predators: an integrative view of escape decisions. Cambridge University Press, 2015.

[2] A. Al-Fuqaha, M. Guizani, M. Mohammadi, M. Aledhari, and M. Ayyash. Internet of things. IC Surveys \& Tutorials, 17(4):2347-2376, 2015.

[3] S. Wu and Y. Tseng. Wireless ad hoc networking. CRC Press, 2007.

[4] P. Jadhav and R. Satao. A survey on opportunistic routing protocols for wsns. ICCCV) 2016.

[5] P. O'Donoghue and C. Rutz. Real-time anti-poaching tags could help prevent imminent species extinctions. Journal of Applied Ecology, 53(1):5-10, 2016.

[6] P. Juang, H. Oki, Y. Wang, M. Martonosi, L. Peh, and D. R. Energy-efficient computing for wildlife tracking. ACM SIGARCH, 30, 2002.

[7] A. Mainwaring, D. Culler, J. Polastre, R. Szewczyk, and J. Anderson. Wsns for habitat monitoring. In IWSN, pages 88-97. Acm, 2002.

[8] S. Raza, P. Misra, Z. He, and T. Voigt. Building the internet of things with bluetooth smart. Elsevier, Ad Hoc Networks, 57:19-31, 2017.

[9] P. Kindt and S. Chakraborty. Neighbor discovery latency in ble-like duty-cycled protocols. CoRR, abs/1509.04366, 2015.

[10] N. Sornin, M. Luis, T. Eirich, T. Kramp, and O. Hersent. Lorawan specifications. LoRa Alliance, 2015.

[11] M. Centenaro, L. Vangelista, A. Zanella, and M. Zorzi. The rising stars in the iot and smart city scenarios. IWC, 23(5):60-67, October 2016.

[12] Anupriya K., Jubin S., J. Yomas, Hari B., and T. Dwarakanath. Integrating zigbee and sub ghz. In $I C$ GET, pages 1-5, Nov 2016.

[13] Sx1276, August 2016. [online] http://www.semtech.com/ wireless-rf/rf-transceivers/sx1276/.

[14] E. D. Ayele, C. Hakkenberg, J. Meijers, K. Zhang, N. Meratnia, and P. J. M. Havinga. Performance analysis of lora radio for an indoor iot applications. In IoTGC, 2017.

[15] E. D. Ayele, N. Meratnia, and P. J. M. Havinga. Hama: A herd-movement adaptive mac protocol for wireless sensor networks. In NTMS, pages 1-7, Nov 2016.

[16] A. Keränen, J. Ott, and T. Kärkkäinen. The one simulator for dtn protocol evaluation. In IC, ICST, 2009.

[17] R. Jovani and R. Mavor. Group size versus individual group size frequency distributions. Animal Behaviour, Elsevier, 82(5):1027 - 1036, 2011. 